\title{
Adaptive power allocation and outage performance of cognitive best relay cooperation systems with multiple primary transceiver pairs and direct path between cognitive source and destination
}

\author{
Xiangdong Jia ${ }^{1,2,3^{*}}$, Ming Zhou' ${ }^{1}$, Xiaochao Dang ${ }^{1}$, Longxiang Yang ${ }^{2,3}$ and Hongbo Zhu ${ }^{2,3}$
}

\begin{abstract}
Based on decode-and-forward (DF) protocol, this work focuses on the adaptive power allocation and outage performance of underlay cognitive radio and opportunistic relaying (UCR-OR) systems with direct path between cognitive source and destination. The UCR-OR systems suffer from the interference of multiple primary user (PU) pairs. Under the outage constraint of PUs and the cognitive peak transmit power limit, we first obtain the adaptive power allocation schemes for secondary transmitters. Secondly, we obtain the exact closed-form expression to the outage probability of UCR-OR systems by using appropriate mathematical proof. Finally, to obtain a clear insight and to highlight the effect of system parameters on the performance of UCR-OR systems, the asymptotic closed-form expression of outage probability is achieved with the assumption of high cognitive transmit power. The presented simulations show that, due to the adaptive power allocation employed, the outage probability of UCR-OR systems is decreasing with PUs' transmit power $P_{p}$ when $P_{p}$ is less than a specific value $P_{P}^{*}$. Only when the value of $P_{p}$ is greater than $P_{P}^{*}$ the outage probability is increasing gradually with the increase of $P_{P}$. When the transmit power of PUs is very high, the outage probability of UCR-OR systems tends to one. That is to say, in this case, the increase of PUs' transmit power degrades severely the performance of UCR-OR systems. Besides this, it is also found that the diversity gain of UCR-OR systems is proportional to the number of cognitive relays. The parameters of PUs only affect the coding gain of UCR-OR but not the diversity gain.
\end{abstract}

Keywords: Cognitive radio; Opportunistic relaying; Direct path; Multiple primary user pairs

\section{Introduction}

Since the electromagnetic spectrum is becoming more and more scarce, improving spectrum efficiency is becoming extremely important for the sustainable development of wireless communication systems and service. However, under the current command-andcontrol spectrum management policy, spectrum resource is not utilized sufficiently as reported by the Federal

\footnotetext{
* Correspondence: jiaxd@nwnu.edu.cn

${ }^{1}$ College of Computer Science and Engineering, Northwest Normal

University, Lanzhou 730070, China

${ }^{2}$ Wireless Communication Key Lab of Jiangsu Province, Nanjing University of

Posts and Telecommunications, Nanjing 210003, China

Full list of author information is available at the end of the article
}

Communications Commission [1] and becomes crowded due to the increasing number of various bandwidthconsuming wireless applications. Recently, cognitive radio (CR) has been proposed as an effective solution to deal with these problems by allowing the access of unlicensed secondary users (SUs) to the frequency band that is allowed to licensed primary users (PUs), in a way that does not affect the quality of service (QoS) of the licensed primary systems $[2,3]$. In general, there are three main $C R$ paradigms: interweave, overlay, and underlay [4]. Among the three paradigms, the underlay paradigm has been considered as a promising solution due to the high spectrum 
efficiency and has become the hot topic of wireless communications [5-7]. The basic idea of underlay CR is that SUs are allowed to share the spectrum with PUs so long as the interference they create on PUs remains below a specific threshold. This results in the improvement on spectrum efficiency. The underlay paradigm is also called spectrum-sharing paradigm $[8,9]$.

However, due to the stringent interference constraint, very low transmit power level is often allowed for the secondary transmitters, and this would significantly degrade the QoS of cognitive systems and reduce the coverage of cognitive networks. One efficient method to improve the performance of cognitive systems is to employ the cooperative communication techniques $[10,11]$. Cooperative communications allow different users in a wireless network to collaborate and share each other's resource; thus, a particular user may transmit data of its own or assist another user through forwarding the received message by acting as a relay. Cooperation among the users helps in generating diversity and enhancing communication coverage [12-14]. Aiming at such improvements, so far, various cooperation schemes have been proposed in literature. Among them, opportunistic relaying (OR) has been shown simple but achieving near optimal outage performance with full diversity. In [15], authors have found that OR schemes can obtain the same diversity order as obtained by the complex distributed space-time code ones. The multi-user multi-relay scenarios have been considered in [16]. The results in [16] showed that the employment of multi-relay can enhance the diversity gain such that the system performance can be improved greatly. Thus, the combination of underlay CR and OR (UCR-OR) can not only undoubtedly inherit the advantages of the two techniques but also shed new light on high performance. For example, [17] is a very important work about cognitive radio with relay cooperation. In this work, authors have presented an exact outage performance analysis for the rates of a decode-and-forward cooperative network where a source communicates with its destination using the well-known repetition-based relaying scheme or using the single best relay, i.e., selection cooperation. Closed-form expressions have been obtained for independent Rayleigh fading channels. The obtained results in [17] indicated that selection cooperation exhibits lower outage probabilities compared to the repetition-based scheme.

Currently, the cognitive radio relay cooperation systems have been investigated widely in literature, see, e.g., [18-23] and references therein. A typical CR relay system consists of a secondary system and a primary system. The primary system includes a pair of primary source and primary destination, while the secondary system includes a secondary source, a secondary destination, and a secondary relay. In such scheme, besides the interference at primary receiver created by SUs, the primary transmitter's interference to the secondary relay and destination cannot be neglected, too. From the viewpoint of SUs, the interference from PUs has severe impact on system performance; thus, it is not ignored and must be considered. With the consideration, based on such system schemes, in $[18,19]$, authors have investigated the outage performance of underlay $\mathrm{CR}$ systems with interference from PUs, where the conventional amplify-and-forward (AF) and decode-and-forward (DF) relaying protocols have been employed, respectively. Though in $[18,19]$ the impact of the primary user's interference on the secondary users for the cognitive systems with single relay has been investigated, the corresponding results for the underlay CR systems with multiple relays have not been presented. That is to say, in the two works, the outage performance of cognitive best relay selection systems has not been studied. Moreover, in $[18,19]$, the direct path transmission between secondary source and destination has been neglected. Thus, in [20], the outage performance of cognitive best relay selection system with primary user interference has been investigated. In particular, authors have obtained the closedform expression for outage probability. The obtained results show that, though the interference from PUs badly degrades the performance of SUs, an increase of relays can compensate the loss. However, the drawback of the work is that the direct path between secondary source and destination has been ignored. In practice, for such scheme investigated in [20], the performance can be further improved by exploiting the direct path between cognitive source and destination. With this consideration, in [21], the outage performance of UCR-OR systems with direct path has been achieved. Though the schemes considered in $[20,21]$ outperform the one in $[18,19]$, the drawback of the schemes in $[20,21]$ is that the maximal transmit power limits at secondary users have been ignored. That is to say, in $[20,21]$, the transmit powers of secondary transmitters were determined only by the interference power constraint at PUs. The available maximal transmit power was assumed to be large enough. However, in practical implementation, the transmitters are maximal power-limited.

In [22], a more general cognitive relay system with primary users' interference has been investigated. In [22], authors have considered a system where the primary system consists of multiple transceiver pairs. This is a realistic consideration in large-scale cognitive systems where the SUs transmit over long distance and may suffer from the interference signals created by multiple primary users. For the scheme, the exact and asymptotic expressions to outage probability were obtained [22]. 
Obviously, in the systems with multiple primary transceivers, the interference at SUs from PUs is increasing with the number of primary transmitters, which degrades greatly the performance of cognitive systems. To compensate the performance loss caused by primary users' interference, as investigated in $[20,21]$, the opportunistic relay schemes should be employed. Therefore, in [23], the outage performance of UCR-OR with multiple primary transceivers has been investigated under imperfect channel state information (CSI). Similarly, as stated in previous, although exploiting the direct path transmission can effectively compensate the performance loss of secondary systems caused by multiple primary users' interference, in [23], the direct link between cognitive source and destination was neglected.

The aforementioned literature review shows that the UCR-OR with multiple primary transceivers is very realistic cognitive radio schemes in large-scale cognitive networks. One example of such cognitive systems is wireless regional area network (WRAN) systems covering a suburb college tower and rural areas. In this case, the cognitive systems would maybe contain multiple PUs and suffer from the interference from multiple PUs. This would degrade greatly the performance of cognitive systems [24]. Therefore, for overcoming this problem, it is an effective solution to compensate the loss by exploiting the multiple relay schemes and the direct path between cognitive source and destination. However, to the best of our knowledge, in existing work about cognitive radio systems, the problem has not been resolved. This paper aims at filling this gap. Particularly, for the UCR-OR systems with multiple primary transceivers and direct path transmission between cognitive source and destination, the peak transmit power constraint and the peak interference power constraint are existent simultaneously. The peak transmit power is the available maximum power of SUs, which is determined by the battery capacity of SUs. In contrast, the peak interference power is the permissible maximum transmission power of SUs in order to guarantee the QoS of primary users. Under the peak interference power constraint, the secondary transmitters should always maintain their transmission power below a predetermined threshold. Obviously, in time-varying channels, it is impossible to satisfy this peak power constraint at all times. For this reason, in this paper, we first consider a constraint based on a stochastic concept instead of the strict peak interference power constraint. The primary systems should be allowed a certain percentage of outage so long as the outage probability of primary systems maintains below a predetermined outage constraint.

The remainder of this paper is organized as follows. In Section 2, the system model and the assumptions are presented. Based on the concept of outage constraint, by using minimum signal-to-interference-plus-noise ratio (SINR) criterion, we present the adaptive power allocation schemes for SUs in Section 3. Section 4 is the outage performance analysis. To highlight the impact of system parameters on performance of UCR-OR systems, in Section 5, the asymptotic outage probability is also derived under the case where the adaptive power allocation is not employed. The simulated results are presented in Section 6. Section 7 is the conclusions.

\section{System model}

As depicted in Figure 1, we consider an UCR-OR system with direct path under peak transmit power and peak interference power constraints. The UCR-OR system suffers from the interference created by multiple PUs. The secondary system is allowed to share the same spectrum band licensed to the primary system. The primary system consists of $M$ primary source $\left(\mathrm{PS}_{m}\right)$ and primary destination $\left(\mathrm{PD}_{m}\right)$ pairs, $m \in\{1, \ldots$, $M\}$, whereas, the secondary system consists of a secondary source (SS), a secondary destination (SD), and $K$ DF secondary relay (SR) $\mathrm{SR}_{k}, k \in \Theta, \Theta=\{1, \ldots, K\}$. This is a realistic consideration in large-scale cognitive systems where the coverage of secondary systems is much larger than that of primary systems. One example of such cognitive systems is WRAN systems covering a suburb college tower and rural areas. In this case, the cognitive systems would maybe contain multiple PUs and suffer from the interference from multiple PUs [24].

It is assumed that all primary and secondary terminals are equipped with single omni-antenna and work on half-duplex mode by using time division multiple access (TDMA). The channel coefficients (or link gains) of SS $\mathrm{SD}, \mathrm{SS}-\mathrm{SR}_{k}, \mathrm{SR}_{k}-\mathrm{SD}$, and $\mathrm{PS}_{m}-\mathrm{PD}_{m}$ communication links are denoted as $\phi, g_{k}, h_{k}$, and $\theta_{m}$, respectively.

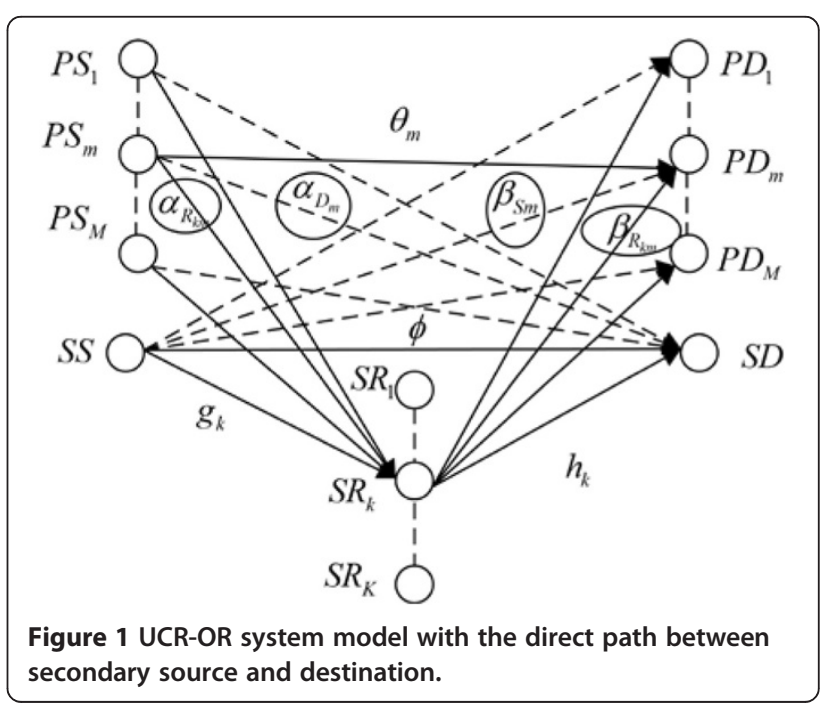


Furthermore, the channel coefficients of the SS - $\mathrm{PD}_{m}$ and $\mathrm{SR}_{k}-\mathrm{PD}_{m}$ interference links are $\beta_{s m}$ and $\beta_{R_{k m}}$, and the ones of the $\mathrm{PS}_{m}-\mathrm{D}$ and $\mathrm{PS}_{m}-\mathrm{SR}_{k}$ interference links are $\alpha_{D m}$ and $\alpha_{R_{k m}}$. We also assume that all channels in each link experience independent and identically distributed (i.i.d) Rayleigh fading. This indicates that for a given link gain $X$, it obeys exponential distribution with hazard rate $1 / \omega_{X}$, denoted by $X \sim \Upsilon\left(1 / \omega_{X}\right)$. Accordingly, as shown in Figure 1, the mean channel powers of $\phi, g_{k}$, $h_{k}, \theta_{m}, \beta_{s m}, \beta_{R_{k m}}, \alpha_{D_{m}}$, and $\alpha_{R_{k m}}$ are $\omega_{\phi}, \omega_{g}, \omega_{h}, \omega_{\theta}, \omega_{\beta_{S}}$, $\omega_{\beta_{R}}, \omega_{\alpha_{D}}$, and $\omega_{\alpha_{R}}$, respectively. At each receiver node, the received signals are affected by symmetry Gaussian additive noise with identical variance $N_{0}$. Note that, for simplicity, we assume that the transmit power of all primary users is $P_{p}$, the one of all secondary relays is $P_{R}$, and the one of secondary source is $P_{s}$. The peak transmit power constraints of secondary source and relays are $P_{S}^{P}$ and $P_{S}^{R}$, respectively.

A whole communication between cognitive source SS and destination SD consists of two phases. In the first phase, the cognitive source SS broadcasts its signal to all relays and destination. For a given relay $\mathrm{SR}_{k}$, the received signal-to-interference-plus-noise radio (SINR) can be formulated as

$$
\gamma_{\mathrm{SR}_{k}}=\frac{P_{S} g_{k}}{\sum_{m=1}^{M} P_{P} \alpha_{R_{k m}}+N_{0}}
$$

Similarly, in this phase the received SINR at cognitive destination SD via direct link is

$$
\gamma_{\mathrm{SD}}=\frac{P_{S} \phi}{\sum_{m=1}^{M} P_{p} \alpha_{D_{m}}+N_{0}}
$$

At the same time, in this phase, the received signal by primary receivers $\mathrm{PD}_{m}$ from $\mathrm{PS}_{m}, m \in\{1, \ldots, M\}$, is corrupted by the interference from the secondary source SS. Thus, combining the received signal from primary transmitter $\mathrm{PS}_{m}$, we also can formulate the SINR at the primary receiver $\mathrm{PD}_{m}$ as

$$
\gamma_{P m}=\frac{P_{P} \theta_{m}}{P_{S} \beta_{S m}+N_{0}}
$$

Since we are concerning a half-duplex two-hop DF relay transmission, in the second phase, the best relay should be selected from decoding subset $\mathrm{DS}_{N}$ that is defined as the subset of $N$ relays able to decode the secondary source's information in the first phase. The selected best relay is given by

$$
b=\arg \underbrace{\max }_{k \in \mathrm{DS}_{N}}\left\{\gamma_{\mathrm{DR}_{k}}\right\}
$$

where the instantaneous SINR $\gamma_{\mathrm{DR}_{k}}$ via random relay $R_{k}$ is defined as

$$
\gamma_{\mathrm{DR}_{k}}=\frac{P_{R} h_{k}}{\sum_{m=1}^{M} P_{P} \alpha_{D m}+N_{0}}
$$

The corresponding instantaneous SINR via the selected best relay $\mathrm{SR}_{b}$ at the cognitive destination $\mathrm{SD}$ is

$$
\gamma_{\mathrm{DR}_{b}}=\underbrace{\max }_{k \in \mathrm{DS}_{N}}\left\{\gamma_{\mathrm{DR}_{k}}\right\}
$$

Thus, combining the received SINRs via direct link and relay link, the total received SINR at destination is

$$
\gamma_{\mathrm{Tot}}=\gamma_{\mathrm{SD}}+\gamma_{\mathrm{DR}_{b}}
$$

Similarly, in this phase, the received expectation signals by primary receivers $\mathrm{PD}_{m}$ are also corrupted by the interference from the selected best relay $\mathrm{SR}_{b}$. The corresponding SINR at $\mathrm{PD}_{m}$ is given by

$$
\gamma_{P_{R_{b m}}}=\frac{P_{P} \theta_{m}}{P_{R} \beta_{R_{b m}}+N_{0}}
$$

where $b$ is defined by Equation 4 .

\section{Outage constraint and adaptive power allocation}

Under the peak transmit power limit, it is impossible to satisfy the interference constraint at all times. For this reason, it makes sense to consider a constraint based on a stochastic concept instead of the power constant. The primary user should be allowed a certain percentage of outage. This yields that the secondary source SS has an adaptive transmit power policy. From the system model given in Figure 1, it is easy to see that, for a give primary transmission $\mathrm{PS}_{m}-\mathrm{PD}_{m}$, the outage constant is given by

$$
\operatorname{Pr}\left\{\gamma_{P m} \leq r_{\text {th }}^{P}\right\} \leq \varepsilon
$$

where $\operatorname{Pr}\{$.$\} demotes probability, \gamma_{P m}$ is defined by Equation 3, $\gamma_{\text {th }}^{P}$ is the outage threshold of primary systems, and $\varepsilon$ is the outage constant of PUs. Obviously, to guarantee that all primary receivers satisfy the outage constraint $\varepsilon$, the minimum SINR selection criterion should be employed. We have

$$
P_{\text {out }}^{p_{1}}=\operatorname{Pr}\{\underbrace{\min }_{m=1, \ldots, M}\left(\gamma_{P m}\right) \leq \gamma_{\text {th }}^{P}\} \leq \varepsilon
$$

By using minimum SINR criterion, Equations 3 and 10 show the relationship between the power $P_{s}$ and outage 
constraint $\varepsilon$ of primary receivers. It is easy to see that the outage probability $P_{\text {out }}^{p_{1}}$ is proportional to $P_{s}$. By taking the maximum value of $P s$, we have $P_{\text {out }}^{p_{1}}=\varepsilon$. With this consideration, we can obtain the maximal permissible transmit power $P_{s}$ of the secondary source SS. Using order statistics [25], we have

$$
P_{\text {out }}^{p_{1}}=\operatorname{Pr}\{\underbrace{\min }_{m=1, \ldots, M}\left(\gamma_{P m}\right) \leq \gamma_{\text {th }}^{P}\}=1-\operatorname{Pr}\{\underbrace{\min }_{m=1, \ldots, M}\left(\gamma_{P m}\right)>\gamma_{\text {th }}^{P}\}
$$

From Equation 3, it is found that the random variables (RVs) $\gamma_{P m}$ are independent mutual. This leads to

$$
\begin{aligned}
P_{\mathrm{out}}^{p_{1}} & =1-\prod_{m=1}^{M} \operatorname{Pr}\left\{\gamma_{P m}>\gamma_{\mathrm{th}}^{P}\right\} \\
& =1-\prod_{m=1}^{M}\left\{1-\operatorname{Pr}\left\{\gamma_{P m} \leq \gamma_{\mathrm{th}}^{P}\right\}\right\}
\end{aligned}
$$

With the definition $\gamma_{P m}$ in Equation 3 and $\theta_{m} \sim \Upsilon(1 /$ $\left.\omega_{\theta}\right)$, having

$$
\operatorname{Pr}\left\{\gamma_{P m} \leq \gamma_{\text {th }}^{P}\right\}=1-\exp \left(-\frac{N_{0} \gamma_{\text {th }}^{P}}{P_{P} \omega_{\theta}}\right) \int_{0}^{\infty} \exp \left(-\frac{P_{S} \gamma_{\text {th }}^{P}}{P_{P} \omega_{\theta}} x\right) f_{\beta_{S m}}(x) d x
$$

where $f_{\beta_{S m}}(\cdot)$ is the PDF of the RV $\beta_{S m}$. By using $\beta_{S m} \sim \gamma$ $\left(1 / \omega_{\beta_{S}}\right)$ and taking the integral of Equation 13 with respect to $\beta_{\text {Sm }}$ yields

$$
\operatorname{Pr}\left\{\gamma_{P m} \leq \gamma_{\text {th }}^{P}\right\}=1-\exp \left(-\frac{N_{0} \gamma_{\text {th }}^{P}}{P_{P} \omega_{\theta}}\right)\left(\frac{P_{S} \omega_{\beta_{S}}}{P_{P} \omega_{\theta}} \gamma_{\text {th }}^{P}+1\right)^{-1}
$$

Combining Equations 14, 12, and 10, the outage constraint of primary receivers is written as

$$
P_{\text {out }}^{p_{1}}=1-\exp \left(-\frac{M N_{0} \gamma_{\text {th }}^{P}}{P_{P} \omega_{\theta}}\right)\left(\frac{P_{S} \omega_{\beta_{S}}}{P_{P} \omega_{\theta}} \gamma_{\text {th }}^{P}+1\right)^{-M} \leq \varepsilon
$$

After some mathematical manipulation, the maximum permissible transmit power $P_{s}$ of secondary source under the outage constraint $\varepsilon$ of primary receivers is given by

$$
P_{S}=X^{P} \frac{P_{P} \omega_{\theta}}{P_{S} \omega_{\beta_{S}}}
$$

Where $X^{P}$ is defined as

$$
X^{P}=\max \left(0,\left(\exp \left(-\left(\frac{N_{0} \gamma_{\text {th }}^{P}}{P_{P} \omega_{\theta}}+\frac{1}{M} \ln (1-\varepsilon)\right)\right)-1\right)\right)
$$

At the same time, the secondary source SS must satisfy the peak transmit power constraint, i.e., $P_{S} \leq P_{S}^{P}$. Thus, the adaptive power allocation policy for secondary source SS is given by

$$
P_{S}=\min \left\{P_{S}{ }^{P}, X^{P} \frac{P_{P} \omega_{\theta}}{P_{S} \omega_{\beta_{S}}}\right\}
$$

In the second phase, based on the selection criterion (Equation 4), the best relay $\mathrm{SR}_{b}$ is selected from decoding subset DS to forward the received signals with power $P_{R}$. Similarly, the transmit power $P_{R}$ must satisfy the outage constraint $\varepsilon$ of primary receivers, which is given by

$$
P_{\text {out }}^{p_{2}}=P_{r}\{\underbrace{\min }_{m=1, \ldots, M}\left(\frac{P_{p} \theta_{m}}{P_{R} \beta_{R_{b m}}+N_{0}}\right) \leq r_{\text {th }}^{P}\} \leq \mathcal{E}
$$

Due to the fact that the selection of the best relay is independent of the link $\mathrm{SR}_{k}-\mathrm{PD}_{m}$, by using the similar method as in Equations 10 to 16 and the peak transmit power $P_{R}^{P}$ the adaptive power allocation policy for the selected best relay $\mathrm{SR}_{b}$ is given by

$$
P_{R}=\min \left\{P_{R}^{P}, X^{P} \frac{\omega_{\theta} P_{P}}{\omega_{\beta_{R}} r_{\text {th }}^{P}}\right\}
$$

where $X^{P}$ is defined by Equation 17 .

Therefore, by using Equations 16 and 20, the transmit powers $P_{s}$ and $P_{R}$ can be determined. Due to the fact that the DF protocol is employed, the power allocation for $P_{s}$ and $P_{R}$ is manipulated separately. For $P_{s}$, the secondary relays and the primary destination send the local CSIs to the secondary source firstly by using feedback links. After collecting the CSIs from the secondary relays and the primary destination, with Equation 16 the transmit power $P_{s}$ of the secondary source can be determined. For $P_{R}$, in our scheme, a distributed scheme is employed, which combines the best relay selection and adaptive power allocation. The basic idea is that each relay sets up an internal timer which triggers transmission. Assuming synchronization among the secondary relays, all secondary relays start their timer simultaneously, whose initial values are inversely proportional to the corresponding SINR given by Equation 5. Since the cognitive destination has the local channel state information (CSI), it can send feedback to the secondary relays. The best relay is the one with its timer reduced to zero first. When the timer of best relay has expired, the relay is expected to broadcast a 'flag' message to neighboring nodes to prevent other relays from transmission. Then, by using the collected CSI from the secondary destination and the primary destination, the selected best relay calculates the transmit power $P_{R}$ according to Equation 20. Obviously, due to the distributed scheme employed, the implementation complexity of the scheme is lower than the centralized scheme. 


\section{Exact outage performance analyses}

In this section, we investigate the outage probability of the considered UCR-OR systems. The outage probability is an effective method to quantify the system performance, which is defined as the probability that the instantaneous end-to-end SNR (or SINR) falls below a predefined threshold. Since we consider a two-hop DF system with direct path transmission between cognitive source and destination, we should start the analysis by studying the decoding subset $\mathrm{DS}_{N}, N=0, \ldots, K$, i.e., the set of $N$ relays able to decode the information transmitted by cognitive source in $\mathrm{SS}-\mathrm{SR}_{k}$ links, where $k \in \Theta$. Obviously, if the decoding subset is empty, i.e., $N=0$, there is no signal transmitted through cognitive relay links. In this case, only the direct path signals are received by cognitive destination SD. This leads to the outage probability of UCR-OR system given by

$$
P_{\text {out }}^{1}=\operatorname{Pr}\left\{\mathrm{DS}_{0}, \gamma_{\mathrm{SD}} \leq \gamma_{\text {th }}^{S}\right\}
$$

where the direct link SINR $\gamma_{\mathrm{SD}}$ is given by Equation 2, and $\gamma_{\text {th }}^{S}$ is the outage threshold at cognitive destination.

On the contrary, if the decoding subset is not empty, i.e., $N \neq 0$, the best relay among the decoding subset $\mathrm{DS}_{N}$ is selected to forward the received source signals. In this case, the cognitive destination SD receives the direct link signals and the best relay link signals, simultaneously. The cognitive destination employs the maximal ratio combiner (MRC) to combine the received signals. According to the total probability theory, the outage probability is given by

$$
P_{\text {out }}^{2}=\sum_{N=1}^{K} \underbrace{\operatorname{Pr}\left\{\mathrm{DS}_{N}\right\}}_{P_{\text {out }}^{21}} \underbrace{\operatorname{Pr}\left\{\gamma_{\mathrm{SD}}+\gamma_{D R_{b}} \leq \gamma_{\text {th }}^{S} \mid \mathrm{DS}_{N}\right\}}_{P_{\text {out }}^{22}}
$$

Finally, combining Equations 21 and 22, the total outage probability of the DF UCR-OR systems can be written as

$$
P_{\text {out }}=P_{\text {out }}^{1}+P_{\text {out }}^{2}
$$

In the following subsection, we would derive the closed-form expressions to $P_{\text {out }}^{1}$ and $P_{\text {out }}^{2}$.

\subsection{Detailed analyses to $P_{\text {out }}^{1}$}

Here, we first derive the closed-form expression to $P_{\text {out }}^{1}$. Due to the fact that the two events $\left\{\mathrm{DS}_{0}\right\}$ and $\left\{\gamma_{\mathrm{SD}} \leq \gamma_{\text {th }}^{S}\right\}$ are independent mutually, we can formulate the outage probability $P_{\text {out }}^{1}$ as

$$
P_{\text {out }}^{1}=\operatorname{Pr}\left\{\mathrm{DS}_{0}\right\} \operatorname{Pr}\left\{\gamma_{\mathrm{SD}} \leq \gamma_{\text {th }}^{S}\right\}
$$

Since $\operatorname{Pr}\left\{\mathrm{DS}_{0}\right\}$ denotes the probability that there is no relay decoding correctly the cognitive source signals in the first phase, using the equivalent SINR (Equation 1) and the fact that $\gamma_{\mathrm{SR}_{k}}$ are independent mutually, we have

$$
\operatorname{Pr}\left\{\mathrm{DS}_{0}\right\}=\prod_{k=1}^{K} \operatorname{Pr}\left\{\frac{P_{S} g_{k}}{Z_{k}+N_{0}} \leq \mu_{\mathrm{th}}^{S}\right\}
$$

where we define $\mu_{\text {th }}^{S}$ as the outage threshold over the cognitive SS - SR $k$ link, and $Z_{k}=\sum_{m=1}^{M} P_{P} \alpha_{R_{k m}}$. Since $\alpha_{R_{k m}}$ are i.i.d Rayleigh fading channel coefficients with variable $\omega_{\alpha_{R}}$, the random variable $Z_{k}$ is a chi-square RV. The corresponding PDF is given by

$$
f_{Z_{k}}(z)=\frac{1}{\Gamma(M)}\left(\frac{1}{P_{p} \omega_{\alpha_{R}}}\right)^{M} z^{M-1} \exp \left(-\frac{z}{\omega_{\alpha_{R}} P_{P}}\right)
$$

where $\Gamma($.$) is the gamma function defined by (8.310.1) in$ [26]. For the convenience of derivation, we define

$$
\Delta=\operatorname{Pr}\left\{\frac{P_{S} g_{k}}{Z_{k}+N_{0}} \leq \mu_{\mathrm{th}}^{S}\right\}
$$

Then, using $g_{k} \sim \Upsilon\left(1 / \omega_{g}\right)$ leads to

$$
\begin{aligned}
\Delta=1-\frac{1}{\Gamma(M)}\left(\frac{1}{P_{p} \omega_{\alpha_{R}}}\right)^{M} & \exp \left(-\frac{\mu_{t h}^{S} N_{0}}{P_{S} \omega_{g}}\right) \int_{0}^{\infty} z^{M-1} \\
& \exp \left(-z\left(\frac{\mu_{t h}^{S}}{P_{S} \omega_{g}}+\frac{1}{\omega_{\alpha_{R}} P_{P}}\right)\right) d z
\end{aligned}
$$

Using (3.351.3) in [26] leads to

$$
\Delta=1-\exp \left(-\frac{\mu_{\mathrm{th}}^{S} N_{0}}{P_{S} \omega_{g}}\right)\left(\frac{P_{p} \omega_{\alpha_{R}}}{P_{S} \omega_{g}} \mu_{\mathrm{th}}^{S}+1\right)^{-M}
$$

Finally, with the consideration that all channels in the cognitive $S S-S R_{k}$ link are i.i.d fading, by substituting Equation 29 into Equation 25, we have

$$
\operatorname{Pr}\left\{\mathrm{DS}_{0}\right\}=\Delta^{K}=\left(1-\exp \left(-\frac{\mu_{\mathrm{th}}^{S} N_{0}}{P_{S} \omega_{g}}\right)\left(\frac{P_{p} \omega_{\alpha_{R}}}{P_{S} \omega_{g}} \mu_{\mathrm{th}}^{S}+1\right)^{-M}\right)^{K}
$$

In Equation 24, the term $\operatorname{Pr}\left\{\gamma_{\mathrm{SD}} \leq \gamma_{\text {th }}^{S}\right\}$ is outage probability over the direct link SS - SD. Observing the definitions $\gamma_{\mathrm{SD}}$ and $\gamma_{\mathrm{SR}_{k}}$ in Equations 2 and 1, we can find that $\gamma_{\mathrm{SD}}$ and $\gamma_{\mathrm{SR}_{k}}$ have similar forms. Therefore, the evaluation to $\operatorname{Pr}\left\{\gamma_{\mathrm{SD}} \leq \gamma_{\text {th }}^{S}\right\}$ can be achieved from Equation 29 through the respective parameter exchange, i.e., $\omega_{\alpha_{R}}->\omega_{\alpha_{D}}, \omega_{g}->\omega_{\phi}$, and $\mu_{\mathrm{th}}^{S}->\gamma_{\mathrm{th}}^{S}$, which is given by 


$$
\operatorname{Pr}\left\{\gamma_{\mathrm{SD}} \leq \gamma_{\mathrm{th}}^{S}\right\}=1-\exp \left(-\frac{\gamma_{\mathrm{th}}^{S} N_{0}}{P_{S} \omega_{\phi}}\right)\left(\frac{P_{p} \omega_{\alpha_{D}}}{P_{S} \omega_{\phi}} \gamma_{\text {th }}^{S}+1\right)^{-M}
$$

Substituting Equations 31 and 30 into Equation 24, the evaluation to $P_{\text {out }}^{1}$ is achieved.

\subsection{Detailed analysis to $P_{\text {out }}^{2}$}

Equation 22 shows that the outage probability $P_{\text {out }}^{2}$ consists of two parts, i.e., $P_{\text {out }}^{21}$ and $P_{\text {out }}^{22}$. The term $P_{\text {out }}^{21}=\operatorname{Pr}$ $\left\{\mathrm{DS}_{N}\right\}$ denotes the probability that $N$ relays out of the $K$ candidates are in the decoding subset DS. Thus, using the equivalent SINR (Equation 1) and the fact that $\gamma_{\mathrm{SR}_{k}}$ are independent mutually, we have

$$
P_{\text {out }}^{21}=\sum_{\mathrm{DS}_{N}} \prod_{k \in \mathrm{DS}_{N}} \operatorname{Pr}\left\{\gamma_{\mathrm{SR}_{k}} \geq \mu_{\text {th }}^{S}\right\} \prod_{j \notin \mathrm{DS}_{N}} \operatorname{Pr}\left\{\gamma_{\mathrm{SR}_{j}} \leq \mu_{\text {th }}^{S}\right\}
$$

Since we are assuming that all channels in each link experience i.i.d Rayleigh fading, the outage probability $P_{\text {out }}^{2}$ does not depend on which relay nodes are in the decoding subset DS, but on how many relay nodes belong to the decoding subset. Therefore, using the definition $\Delta=\operatorname{Pr}\left\{\gamma_{\mathrm{SR}_{k}} \leq \mu_{\text {th }}^{S}\right\}$ and the result given by Equation 29, it is easy to see that $P_{\text {out }}^{21}$ is given by

$$
P_{\text {out }}^{21}=C_{N}^{K}(1-\Delta)^{N} \Delta^{K-N}
$$

At the same time, Equation 22 shows that, to obtain the evaluation of $P_{\text {out }}^{2}$, the term $P_{\text {out }}^{22}=\operatorname{Pr}$ $\left\{\gamma_{\mathrm{SD}}+\gamma_{\mathrm{DR}_{b}} \leq \gamma_{\text {th }}^{S} \mid \mathrm{DS}_{N}\right\}$ is required. Using $\gamma_{\mathrm{SD}}$ and $\gamma_{\mathrm{DR}_{b}}$ defined by Equations 2 and $6, P_{\text {out }}^{22}$ can be formulated as

$$
P_{\text {out }}^{22}=\operatorname{Pr}\left\{\left(\frac{P_{S} \phi}{Y+N_{0}}+\frac{Z}{Y+N_{0}}\right) \leq \gamma_{\text {th }}^{S}\right\}
$$

where we define $Y=\sum_{m=1}^{M} P_{P} \alpha_{D_{m}}$ and $Z=\underbrace{\max }_{k \in \mathrm{DS}}\left\{P_{R} h_{k}\right\}$. Due to the correlation among the received SINR at cognitive relays and destination caused by PUs' interference, the exact closed-form expression to this cannot be calculated as the conventional analysis any more. Therefore, conditioned on $Y$ and $Z, P_{\text {out }}^{22}$ is given by
$P_{\text {out }}^{22}=\int_{0}^{\infty} \int_{\frac{z}{\gamma_{\text {th }}^{S}}-\infty}^{\infty} \operatorname{Pr}\left(\phi \leq \frac{y+N_{0}}{P_{S}}\left(\gamma_{\text {th }}^{S}-\frac{z}{y+N_{0}}\right) \mid y, z\right) f_{Y}(y) f_{Z}(z) d y d z$

Since the PDF of $Y=\sum_{m=1}^{M} P_{P} \alpha_{D_{m}}$ can be obtained from Equation 26 through the respective parameter exchange $\omega_{\alpha_{R}}->\omega_{\alpha_{D}}$, with $\phi \sim \Upsilon\left(1 / \omega_{\phi}\right)$, we have $P_{\text {out }}^{22}$ given by

$$
P_{\text {out }}^{22}=P_{\text {out }}^{22-1}-P_{\text {out }}^{22-2}
$$

where the two parts $P_{\text {out }}^{22-1}$ and $P_{\text {out }}^{22-2}$ are given by

$$
\begin{aligned}
P_{\text {out }}^{22-1}=\frac{1}{\Gamma(M)}\left(\frac{1}{P_{P} \omega_{\alpha_{D}}}\right)^{M} \int_{0}^{\infty} \int_{\frac{z}{\gamma_{\text {th }}^{S}}-N_{0}}^{\infty} y^{M-1} \\
\\
\exp \left(-\frac{y}{P_{p} \omega_{\alpha_{D}}}\right) f_{Z}(z) d y d z
\end{aligned}
$$

$$
\begin{aligned}
P_{\text {out }}^{22-2}= & \frac{1}{\Gamma(M)}\left(\frac{1}{P_{P} \omega_{\alpha_{D}}}\right)^{M} \int_{0}^{\infty} \int_{\frac{z}{\gamma_{\text {th }}^{S}}-N_{0}}^{\infty} \\
& \exp \left(-\frac{1}{\omega_{\phi}} \frac{y+N_{0}}{P_{S}}\left(\gamma_{\text {th }}^{S}-\frac{z}{y+N_{0}}\right)\right) y^{M-1} \\
& \exp \left(-\frac{y}{P_{p} \omega_{\alpha_{D}}}\right) f_{Z}(z) d y d z
\end{aligned}
$$

We first consider the part $P_{\text {out }}^{22-2}$, using $\int_{u}^{\infty} x^{\nu-1} \exp (-\mu x)$ $d x=\mu^{-v} \Gamma(\nu, \mu u)$ given by (3.381.3) in [26], it can be written as

$$
\begin{aligned}
& P_{\text {out }}^{22-2}=\frac{1}{\Gamma(M)} \exp \left(-\frac{N_{0} \gamma_{\text {th }}^{S}}{P_{S} \omega_{\phi}}\right)\left(\frac{1}{P_{P} \omega_{\alpha_{D}}}\right)^{M} \\
& \times \int_{0}^{\infty} \exp \left(\frac{z}{P_{S} \omega_{\phi}}\right)\left(\frac{\gamma_{\text {th }}^{S}}{P_{S} \omega_{\phi}}+\frac{1}{P_{p} \omega_{\alpha_{D}}}\right)^{-M} \\
& \Gamma\left(M,\left(\frac{z}{\gamma_{\text {th }}^{S}}-N_{0}\right)\left(\frac{\gamma_{\text {th }}^{S}}{P_{S} \omega_{\phi}}+\frac{1}{P_{p} \omega_{\alpha_{D}}}\right)\right) f_{Z}(z) d z
\end{aligned}
$$

where $\Gamma(.,$.$) is the incomplete gamma function defined$ by (8.350.2) in [26]. Using the identity $\Gamma(n+1, x)=n$ ! $\exp (-x) \sum_{l=0}^{n} \frac{x^{l}}{l !}$ yields 


$$
\begin{aligned}
P_{\text {out }}^{22-2}=\left(\frac{1}{P_{P} \omega_{\alpha_{D}}}\right)^{M} \exp \left(\frac{N_{0}}{P_{p} \omega_{\alpha_{D}}}\right) \int_{0}^{\infty} \exp \left(-\frac{z}{P_{p} \omega_{\alpha_{D}} \gamma_{\text {th }}^{S}}\right) \sum_{n_{1}=0}^{M-1} \frac{1}{n_{1} !} \\
\left(\frac{z}{\gamma_{\text {th }}^{S}}-N_{0}\right)^{n_{1}}\left(\frac{\gamma_{\text {th }}^{S}}{P_{S} \omega_{\phi}}+\frac{1}{P_{p} \omega_{\alpha_{D}}}\right)^{-M+n_{1}} f_{Z}(z) d z
\end{aligned}
$$

Using the binomial expansion, having

$$
\begin{aligned}
P_{\text {out }}^{22-2}= & \left(\frac{1}{P_{P} \omega_{\alpha_{D}}}\right)^{M} \exp \left(\frac{N_{0}}{P_{p} \omega_{\alpha_{D}}}\right) \sum_{n_{1}=0}^{M-1} \frac{\left(-N_{0}\right)^{n_{1}}}{n_{1} !}\left(\frac{\gamma_{\text {th }}^{S}}{P_{S} \omega_{\phi}}+\frac{1}{P_{p} \omega_{\alpha_{D}}}\right)^{-M+n_{1}} \\
& \sum_{n_{2}=0}^{n_{1}}\left(\begin{array}{l}
n_{1} \\
n_{2}
\end{array}\right)(-1)^{n_{2}}\left(\frac{1}{N_{0} \gamma_{\text {th }}^{S}}\right)^{n_{2}} \int_{0}^{\infty} z^{n_{2}} \exp \left(-\frac{z}{P_{p} \omega_{\alpha_{D}} \gamma_{\text {th }}^{S}}\right) f_{Z}(z) d z
\end{aligned}
$$

where $\left(\begin{array}{l}n_{1} \\ n_{2}\end{array}\right)=n_{1} ! /\left(n_{2} !\left(n_{1}-n_{2}\right) !\right)$ is the binomial coefficient. With the definition $Z=\underbrace{\max }_{i \in \mathrm{DS}_{N}}\left\{P_{R} h_{i}\right\}$, it is easy to see that the PDF of the RV $Z$ is given by

$$
f_{Z}(z)=\frac{N}{P_{R} \omega_{h}} \sum_{n_{3}=0}^{N-1}\left(\begin{array}{c}
N-1 \\
n_{3}
\end{array}\right)(-1)^{n_{3}} \exp \left(-\frac{\left(n_{3}+1\right) z}{P_{R} \omega_{h}}\right)
$$

Substituting Equation 42 into Equation 41 yields

$$
\begin{gathered}
P_{\text {out }}^{22-2}=\left(\frac{1}{P_{P} \omega_{\alpha_{D}}}\right)^{M} \exp \left(\frac{N_{0}}{P_{p} \omega_{\alpha_{D}}}\right) \sum_{n_{1}=0}^{M-1} \frac{\left(-N_{0}\right)^{n_{1}}}{n_{1} !} \\
\left(\frac{\gamma_{\text {th }}^{S}}{P_{S} \omega_{\phi}}+\frac{1}{P_{p} \omega_{\alpha_{D}}}\right)^{-M+n_{1}} \sum_{n_{2}=0}^{n_{1}}\left(\begin{array}{c}
n_{1} \\
n_{2}
\end{array}\right)(-1)^{n_{2}}\left(\frac{1}{N_{0} \gamma_{\text {th }}^{S}}\right)^{n_{2}} \\
\times \frac{N}{P_{R} \omega_{h}} \sum_{n_{3}=0}^{N-1}\left(\begin{array}{c}
N-1 \\
n_{3}
\end{array}\right)(-1)^{n_{3}} \int_{0}^{\infty} z^{n_{2}} \\
\exp \left(-z\left(\frac{1}{P_{p} \omega_{\alpha_{D}} \gamma_{\text {th }}^{S}}+\frac{\left(n_{3}+1\right)}{P_{R} \omega_{h}}\right)\right) d z
\end{gathered}
$$

Using (3.351.3) [26], after some mathematical manipulation, the evaluation to $P_{\text {out }}^{22-2}$ is given by

$$
\begin{aligned}
& P_{\text {out }}^{22-2}=\left(\frac{1}{P_{P} \omega_{\alpha_{D}}}\right)^{M} \exp \left(\frac{N_{0}}{P_{p} \omega_{\alpha_{D}}}\right) \sum_{n_{1}=0}^{M-1} \sum_{n_{2}=0}^{n_{1}} \sum_{n_{3}=0}^{N-1}\left(\begin{array}{l}
n_{1} \\
n_{2}
\end{array}\right) \\
&\left(\begin{array}{c}
N-1 \\
n_{3}
\end{array}\right) \frac{(-1)^{n_{1}+n_{2}+n_{3}} N_{0} n_{1} n_{2} !}{n_{1} !} \\
& \times \frac{N}{P_{R} \omega_{h}}\left(\frac{\gamma_{\mathrm{th}}^{S}}{P_{S} \omega_{\phi}}+\frac{1}{P_{p} \omega_{\alpha_{D}}}\right)^{-M+n_{1}}\left(\frac{1}{N_{0} \gamma_{\mathrm{th}}^{S}}\right)^{n_{2}} \\
&\left(\frac{1}{P_{p} \omega_{\alpha_{D}} \gamma_{\mathrm{th}}^{S}}+\frac{\left(n_{3}+1\right)}{P_{R} \omega_{h}}\right)^{-n_{2}-1}
\end{aligned}
$$

For the part $P_{\text {out }}^{22-1}$, using the $\int_{u}^{\infty} x^{\nu-1} \exp (-\mu x) d x=\mu^{-v}$ $\Gamma(v, \mu u)$ given by (3.381.3) in [26] leads to

$$
P_{\text {out }}^{22-1}=\frac{1}{\Gamma(M)} \int_{0}^{\infty} \Gamma\left(M, \frac{1}{P_{p} \omega_{\alpha_{D}}}\left(\frac{z}{\gamma_{\text {th }}^{S}}-N_{0}\right)\right) f_{Z}(z) d y d z
$$

Similar to Equation 40, having

$$
\begin{array}{r}
P_{\text {out }}^{22-1}=\exp \left(\frac{N_{0}}{P_{p} \omega_{\alpha_{D}}}\right) \sum_{m_{1}=0}^{M-1} \frac{1}{m_{1} !}\left(\frac{1}{P_{p} \omega_{\alpha_{D}}}\right)^{m_{1}} \int_{0}^{\infty}\left(\frac{z}{\gamma_{\text {th }}^{S}}-N_{0}\right)^{m_{1}} \\
\exp \left(-\frac{1}{P_{p} \omega_{\alpha_{D}}} \frac{z}{\gamma_{\text {th }}^{S}}\right) f_{Z}(z) d z
\end{array}
$$

Substituting Equation 42 into Equation 46, $P_{\text {out }}^{22-1}$ is written as

$$
\begin{aligned}
P_{\text {out }}^{22-1}= & \exp \left(\frac{N_{0}}{P_{p} \omega_{\alpha_{D}}}\right) \sum_{m_{1}=0}^{M-1} \frac{1}{m_{1} !}\left(\frac{1}{P_{p} \omega_{\alpha_{D}}}\right)^{m_{1}} \frac{N}{P_{R} \omega_{h}} \sum_{m_{2}=0}^{N-1} \\
& \left(\begin{array}{l}
N-1 \\
m_{2}
\end{array}\right)(-1)^{m_{2}}\left(-N_{0}\right)^{m_{1}} \sum_{m_{3}=0}^{m_{1}}\left(\begin{array}{l}
m_{1} \\
m_{3}
\end{array}\right)\left(\frac{-1}{N_{0} \gamma_{\text {th }}^{S}}\right)^{m_{3}} \\
& \times \int_{0}^{\infty} z^{m_{3}} \exp \left(-z\left(\frac{1}{P_{p} \omega_{\alpha_{D}} \gamma_{\text {th }}^{S}}-\frac{\left(m_{2}+1\right)}{P_{R} \omega_{h}}\right)\right) d z
\end{aligned}
$$

Using (3.351.3) in [26], we have

$$
\begin{aligned}
P_{\text {out }}^{22-1}=\exp & \left(\frac{N_{0}}{P_{p} \omega_{\alpha_{D}}}\right) \frac{N}{P_{R} \omega_{h}} \sum_{m_{1}=0}^{M-1} \sum_{m_{2}=0}^{N-1} \sum_{m_{3}=0}^{m_{1}}\left(\begin{array}{l}
N-1 \\
m_{2}
\end{array}\right) \\
& \left(\begin{array}{l}
m_{1} \\
m_{3}
\end{array}\right)(-1)^{m_{1}+m_{2}+m_{3}} \frac{m_{3} ! N_{0} m_{1}}{m_{1} !}\left(\frac{1}{P_{p} \omega_{\alpha_{D}}}\right)^{m_{1}} \\
& \left(\frac{1}{N_{0} \gamma_{\text {th }}^{S}}\right)^{m_{3}}\left(\frac{1}{P_{p} \omega_{\alpha_{D}} \gamma_{\text {th }}^{S}}+\frac{\left(m_{2}+1\right)}{P_{R} \omega_{h}}\right)^{-m_{3}-1}
\end{aligned}
$$

Combining Equations 44 and 48, the result for $P_{\text {out }}^{22}$ can be obtained. Then, by substituting Equations 36 and 33 into Equation 22, we can obtain the closed-form solution to $P_{\text {out }}^{2}$ that is outage probability of DF UCR-OR systems when the decoding subset is not empty.

\section{Asymptotic outage performance analyses}

Although in Section 4 we obtain the exact closed-form expression of outage probability for the considered UCR-OR systems, the derivations are computationally complicated and do not offer insight into the impact of system parameters on system performance. Therefore, in practice, some simplified expressions are required. To this end, we now derive the asymptotic closed-form expressions of outage probability by using the assumption that the value of the transmit power $P_{s}$ is high. At the same time, for simplicity, we also assume $P_{R}=\lambda P_{S}$. Then, from the asymptotic results in high transmit power $P_{s}$, the diversity and coding gains can be achieved. 
For the convenience of derivation, we combine the general expressions 21 and 22 of outage probability and rewrite as

$$
P_{\text {out }}^{\text {Asy }}=\sum_{N=0}^{K} \underbrace{\operatorname{Pr}\left\{\mathrm{DS}_{N}\right\}}_{P_{\text {out }}^{21}} \underbrace{\operatorname{Pr}\left\{\gamma_{\mathrm{SD}}+\gamma_{\mathrm{DR}_{b}} \leq \gamma_{\text {th }}^{S} \mid \mathrm{DS}_{N}\right\}}_{P_{\text {out }}^{22}}
$$

where $P_{\text {out }}^{21}$ and $P_{\text {out }}^{22}$ are defined by Equations 33 and 34, respectively. Equation 33 indicates that the simplified expression for $\Delta$ is required firstly. Using the definition of $\Delta$ and Equation 27, we have

$$
\Delta=\operatorname{Pr}\left\{\frac{P_{S} g_{k}}{Z_{k}+N_{0}} \leq \mu_{\text {th }}^{S}\right\} \approx \operatorname{Pr}\left\{P_{S} g_{k} \leq \mu_{\text {th }}^{S} Z_{k}\right\}
$$

With $g_{k} \sim \Upsilon\left(1 / \omega_{g}\right)$ and the assumption of high $P_{s}$, having

$$
\Delta \approx \frac{\mu_{t h}^{S}}{P_{S} \omega_{g}} \int_{0}^{\infty} z f_{Z_{k}}(z) d z
$$

Substituting the PDF (Equation 26) of $Z_{k}$ into Equation 51 leads to

$$
\begin{aligned}
\Delta & \approx \frac{1}{\Gamma(M)} \frac{\mu_{\mathrm{th}}^{S}}{P_{S} \omega_{g}}\left(\frac{1}{P_{p} \omega_{\alpha_{R}}}\right)^{M} \int_{0}^{\infty} z^{M} \exp \left(-\frac{z}{\omega_{\alpha_{R}} P_{P}}\right) d z \\
& =\frac{P_{p} \omega_{\alpha_{R}}}{P_{S} \omega_{g}} M \mu_{\mathrm{th}}^{S}
\end{aligned}
$$

Thus, substituting Equation 52) into Equation 33, the asymptotic expression of $P_{\text {out }}^{21}$ is given by

$$
P_{\mathrm{out}}^{21} \approx C_{N}^{K}\left(\frac{P_{p} \omega_{\alpha_{R}}}{P_{S} \omega_{g}} M \mu_{\text {th }}^{S}\right)^{K-N}
$$

Note that here, we employ the fact $1-\Delta \approx 1$ in high $P_{s}$. For the term $P_{\text {out }}^{22}$ defined by Equation 34, in high transmit power $P_{s}$, we have

$$
P_{\text {out }}^{22} \approx \operatorname{Pr}\left\{P_{S} \phi \leq \gamma_{\text {th }}^{S} Y-Z\right\}
$$

With the definition $Z=\underbrace{\max }_{k \in \mathrm{DS}_{N}}\left\{P_{R} h_{k}\right\}$ and $P_{R}=\lambda P_{S}$, in high transmit power $P_{s}$, the PDF of the random variable $Z$ is given approximately by $f_{Z}(z) \approx \frac{N}{\left(\omega_{h} P_{R}\right)^{N}} z^{N-1}$. This leads to Equation 54 given asymptotically by

$$
\begin{aligned}
P_{\text {out }}^{22} \approx & \frac{1}{P_{S} \omega_{\phi}} \int_{0}^{\infty} \int_{0}^{\gamma_{\mathrm{th}}^{S} y}\left(\gamma_{\mathrm{th}}^{S} y-z\right) f_{Z}(z) f_{Y}(y) d z d y \\
& \approx \frac{1}{P_{S} \omega_{\phi}} \frac{N}{\left(\omega_{h} P_{R}\right)^{N}} \int_{0}^{\infty} \int_{0}^{\gamma_{\mathrm{th}}^{S} y}\left(\gamma_{\mathrm{th}}^{S} y-z\right) z^{N-1} f_{Y}(y) d z d y \\
& =\frac{1}{P_{S} \omega_{\phi}} \frac{1}{\left(\omega_{h} P_{R}\right)^{N}} \frac{1}{N+1} \int_{0}^{\infty}\left(\gamma_{\mathrm{th}}^{S} y\right)^{N+1} f_{Y}(y) d y
\end{aligned}
$$

The PDF of the random variable $Y=\sum_{m=1}^{M} P_{P} \alpha_{D_{m}}$ can be obtained from Equation 26 through the respective parameter exchange $\omega_{\alpha_{R}}->\omega_{\alpha_{D}}$. Thus, Equation 55 is written as

$$
\begin{gathered}
P_{\text {out }}^{22} \approx \frac{1}{P_{S} \omega_{\phi}} \frac{1}{\left(P_{R} \omega_{h}\right)^{N}} \frac{1}{\left(P_{p} \omega_{\alpha_{D}}\right)^{M}} \frac{1}{(N+1) \Gamma(M)}\left(\gamma_{\text {th }}^{S}\right)^{N+1} \int_{0}^{\infty} y^{M+N} \\
\exp \left(-\frac{y}{\omega_{\alpha_{D}} P_{P}}\right) d y \\
=\frac{1}{P_{S} \omega_{\phi}} \frac{\left(P_{P} \omega_{\alpha_{D}}\right)^{N+1}}{\left(P_{R} \omega_{h}\right)^{N}} \frac{(M+N) !}{(N+1) \Gamma(M)}\left(\gamma_{\text {th }}^{S}\right)^{N+1}
\end{gathered}
$$

Finally, substituting Equations 56 and 53 into Equation 49 yields that in high transmit power $P_{s}$, the asymptotic expression of outage probability is given by

$$
\begin{aligned}
P_{\mathrm{out}}^{\mathrm{Asy}} \approx \sum_{N=0}^{K} C_{N}^{K} & \left(\frac{P_{p} \omega_{\alpha_{R}}}{P_{S} \omega_{g}} M \mu_{\mathrm{th}}^{S}\right)^{K-N} \frac{1}{P_{S} \omega_{\phi}} \frac{\left(P_{P} \omega_{\alpha_{D}}\right)^{N+1}}{\left(P_{R} \omega_{h}\right)^{N}} \frac{(M+N) !}{(N+1) \Gamma(M)}\left(\gamma_{\mathrm{th}}^{S}\right)^{N+1} \\
= & \frac{\left(P_{p}\right)^{K+1}}{\left(P_{S}\right)^{K+1}} \frac{1}{\Gamma(M)} \frac{1}{\omega_{\phi}} \sum_{N=0}^{K} C_{N}^{K}\left(\frac{\omega_{\alpha_{R}}}{\omega_{g}}\right)^{K-N} \\
& \frac{\left(\omega_{\alpha_{D}}\right)^{N+1}}{\left(\lambda \omega_{h}\right)^{N}} \frac{(M+N) !}{(N+1)}\left(M \mu_{\mathrm{th}}^{S}\right)^{K-N}\left(\gamma_{\mathrm{th}}^{S}\right)^{N+1}
\end{aligned}
$$

When $\mu_{\text {th }}^{S}=\gamma_{\text {th }}^{S}$, having

$$
P_{\text {out }}^{\text {Asy }} \approx \varepsilon \frac{\left(\gamma_{\text {th }}^{S}\right)^{K+1}}{\left(P_{S}\right)^{K+1}}
$$

Where we define

$\varepsilon=\frac{\left(P_{p}\right)^{K+1}}{\Gamma(M)} \frac{1}{\omega_{\phi}} \sum_{N=0}^{K} C_{N}^{K}\left(\frac{\omega_{\alpha_{R}}}{\omega_{g}}\right)^{K-N} \frac{\left(\omega_{\alpha_{D}}\right)^{N+1}}{\left(\lambda \omega_{h}\right)^{N}} \frac{(M+N) !}{(N+1)}(M)^{K-N}$

Thus, in high transmit power $P_{s}$, the diversity gain is

$$
G_{d}=\lim _{P_{S^{-}}>\infty}-\frac{\log P_{\text {out }}^{\text {Asy }}}{\log P_{S}}=K+1
$$

The coding gain is 


$$
G_{c}=\frac{\varepsilon^{\frac{-1}{K+1}}}{\gamma_{\mathrm{th}}^{S}}
$$

It can be observed from Equations 60 and 61 that the diversity gain of the considered UCR-OR systems is determined by the number of relays, i.e., $G_{d}=K+1$. The parameters of primary system only affect the coding gain, not the diversity gain. This is due to the fact that the key idea of relay cooperation is that multiple single antenna relays work together and form virtual multipleinput and multiple-output (MIMO) systems. In such virtual MIMO systems, the number of the available source-relay or relay-destination transmissions dominates the diversity gain. However, from Equation 3 to 7 , we can find that the mutual interference between PUs and SUs only affects the equivalent SINR of each single path signal but not cause the increase or decrease in the number of the available source-relay or relay-destination transmissions. That is to say, the number of multiple path signals is still $K+1$. Therefore, we have the result that the diversity gain of the UCR-OR systems is $K+1$.

\section{Simulation results and performance comparison analyses}

In previous sections, we obtain the adaptive power allocation schemes of cognitive transmitters for the considered UCR-OR systems under outage and peak transmit power constraints. Based on the results, we achieve the exact evaluation to the outage probability of UCR-OR systems with the multiple PU pairs and the direct path transmission between cognitive source and destination. At the same time, to obtain the insight about the effect of system parameters on outage performance, with the assumption of high transmit power $P_{s}$, the asymptotic closed-form expression of outage probability is derived too. With these derivations, the simulated and numerical results are presented in this section, which is used to validate the derivations and to obtain the acknowledgement about the impact of system parameter on the UCR-OR systems. During the analyses, we use MATLAB to build simulations. In all case, the channels are generated by using MATLAB toolbox 'Rayleigh', which makes a fading channel. Specially, the following system parameters are employed: number of the cognitive relays $K=10$, PUs' outage threshold $\gamma_{\text {th }}^{P}=-9 d B$, mean power of PUs channels $\omega_{\theta}=2$, peak transmit power constraints at SUs $P_{S}^{P}=P_{R}^{P}=10 \mathrm{~dB}$, and noise variance $N_{0}=1$. Note that, for the clarity of comparison analyses, in the sequence discussion, the outage probabilities of the UCR-OR systems with and without direct path are presented simultaneously, which are marked by 'Dir link' and 'No-dir link', respectively.
By taking the channel mean powers $\omega_{g}=\omega_{h}=2$ and $\omega_{\beta_{S}}=\omega_{\beta_{R}}=\omega_{\alpha_{R}}=\omega_{\alpha_{D}}=0.2$ and the outage threshold $\gamma_{\text {th }}^{S}=\mu_{\text {th }}^{S}=-10 d B$, we first investigate the adaptive power allocations for $P_{s}$ and $P_{R}$. Due to the fact that the symmetric system parameters are employed, according to Equations 18 and 20, we have $P_{s}=P_{R}$. Thus, we only present the investigation on $P_{s}$. For $P_{R}$, the results are straightforward. In Figure 2, we present the power $P_{s}$ versus the peak power $P_{P}(d B)$ of primary transmitters under different values of $M$ and $\varepsilon$. The presented figures show clearly that the transmit power $P_{s}$ is changing with $M$ and $\varepsilon$. We first analyze the power allocation of the UCR-OR systems with $M=4$ and $\varepsilon=0.01$. It is observed that $P_{s}=0$ when the value of $P_{p}$ is less than or equal to the specific value $P_{P}^{*}=14 d B$. When $P_{p}$ is greater than $14 d B$, the transmission power $P_{s}$ increases gradually. The transmit power $P_{s}$ ascends to the maximal value as $P_{p}$ increases to another specific value $P_{P}^{* *}=17.5 \mathrm{~dB}$ and then remains constant as $P_{P}>17.5 \mathrm{~dB}$. This can be explained by the fact that the outage probability of PUs is greater than the outage constraint $\varepsilon$ when $P_{P}<14 \mathrm{~dB}$. According to the adaptive power allocation policy, we have $P_{s}=0$. Then, when $P_{P}>14 d B$, the outage probability of PUs satisfies the outage constraint $\varepsilon$. In this case, the increasing $P_{p}$ leads to an increase in the transmission rate of PUs. Accordingly, the secondary transmitters can increase their transmit powers $P_{s}$ and $P_{R}$ and still keep the outage probability of PUs below the given outage constraint $\varepsilon$. Furthermore, as $P_{p}$ continuously increases, the transmit power of SUs would approach the maximal values, $P_{S}^{P}$ and $P_{R}^{P}$, given by Equations 18 and 20, i.e., the performance of the secondary systems is optimal. When

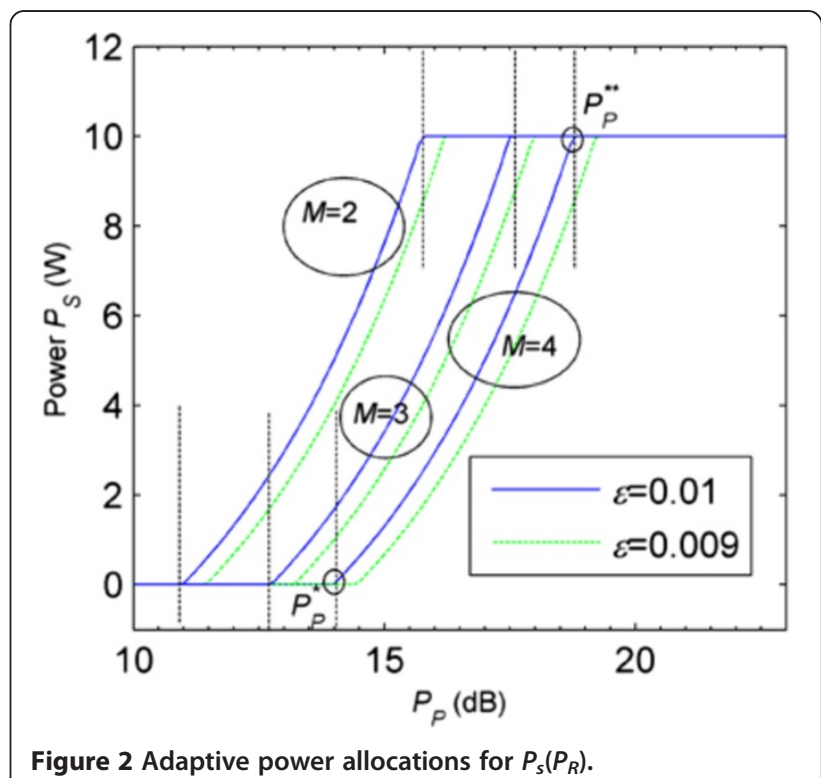


the transmit power of primary users is increased beyond the optimal value $P_{P}^{* *}, P_{s}$ cannot be further increased due to the peak transmit power constraint, i.e., $P_{S}=P_{S}^{P}$. At the same time, the figure also shows that the two specific values $P_{P}^{*}$ and $P_{P}^{* *}$ are different for different values of $M$ and $\varepsilon$.

Figure 3a,b shows the outage probability of UCR-OR systems versus the peak power $P_{P}$ by taking $M=3$ and $\varepsilon=0.01$. In Figure 3a, with the given outage thresholds $\mu_{\mathrm{th}}^{S}=\gamma_{\mathrm{th}}^{S}=-10 \mathrm{~dB}$, we investigate the impact of the mean channel power $\omega_{\phi}$ of direct path SS - SD on the outage probability. The presented results show firstly that the simulations match well with the analytical results, which validate the derivations. At the same time, it is seen that the outage probability of the UCROR systems is decreasing when the value of $P_{p}$ is less than $17.5 d B$. However, it is increasing gradually when $P_{P}$ is greater than $17.5 \mathrm{~dB}$. Similar to Figure 2, this can be explained by the fact the increasing $P_{P}$ leads to an increase in transmission rate of PUs, i.e., improvement in the performance of primary systems. Therefore, the secondary transmitters can increase their transmit power and still keep the outage probability of PUs below the given outage constraint $\varepsilon$. Furthermore, with the continuous increase of $P_{P}$ the transmit power $P_{S}$ and $P_{R}$ of cognitive system will approach the maximal values according to the adaptive power allocation (Equations 18 and 20), i.e., the performance of cognitive systems approaching optimal point. However, if the transmit power $P_{P}$ of PUs is increased beyond the optimal value, the transmit $P_{S}$ and $P_{R}$ of cognitive systems cannot be further increased due to the peak power constraint. Therefore, in this case, any increase in $P_{P}$ will lead to degradation in the performance of the interested UCR-OR systems.

For the impact of the direct link, we can find that the systems with direct link outperform the ones without direct link. With the increase of the direct link mean power $\omega_{\phi}$, the gap between the outage probabilities of the two systems is increasing. For example, when $\omega_{\phi}=0.05$, we can find the gap of outage probabilities is very little and can be ignored. Whereas, when $\omega_{\phi}=1.5$, the outage performance of UCR-OR systems is improved greatly. In this case, the direct link between cognitive source and destination should be considered, which results in the enhancement of communication reliability.

In Figure 3b, by using $\omega_{\phi}=0.5$ and $\gamma_{\text {th }}^{S}=-10 d B$, we investigate the impact of the outage threshold $\mu_{\text {th }}^{S}$ of the first hop. In the figure, we take $\mu_{\text {th }}^{S}=-2,-8$, and-18 $d B$, respectively. It can be clearly seen that the outage probability of the UCR-OR systems is decreasing with the decrease of the outage threshold $\mu_{\mathrm{th}}^{S}$. This is due to the fact that the number of relays in the decoding subset DS is increased as the outage threshold $\mu_{\text {th }}^{S}$ of the first hop is decreased. As a result, the outage performance of the UCR-OR systems is improved. At the same time, it can be also seen that the gap of the outage probabilities between the two UCR-OR systems (with and without direct path) is increasing with the decrease of the outage threshold $\mu_{\text {th }}^{S}$. The observation is explained as follows. As aforementioned, the

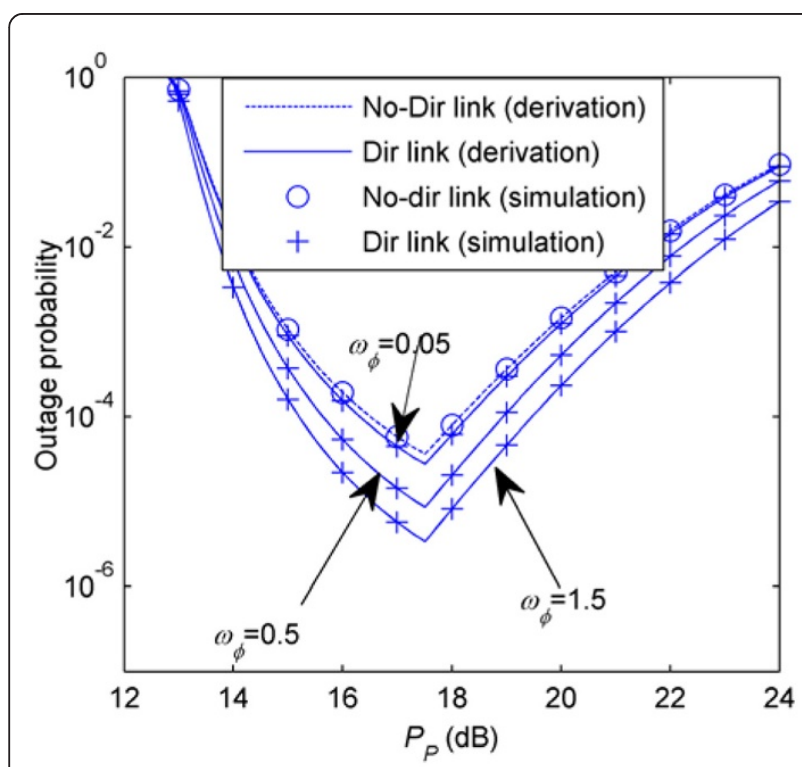

(a)

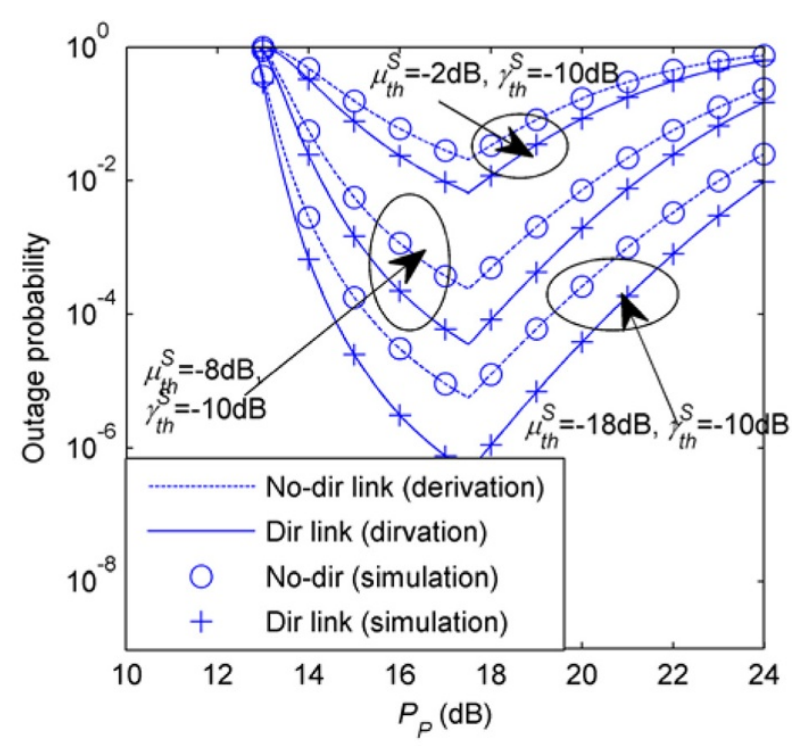

(b)

Figure 3 The impact of the cognitive direct link and the outage threshold $\mu_{t h}^{S}$ on outage performance. (a) Impact of $\omega_{\varphi}$ and (b) impact of outage thresholds $\mu_{\mathrm{th}}^{\mathrm{s}}$. The arrows denote the corresponding curves for given system parameters. 


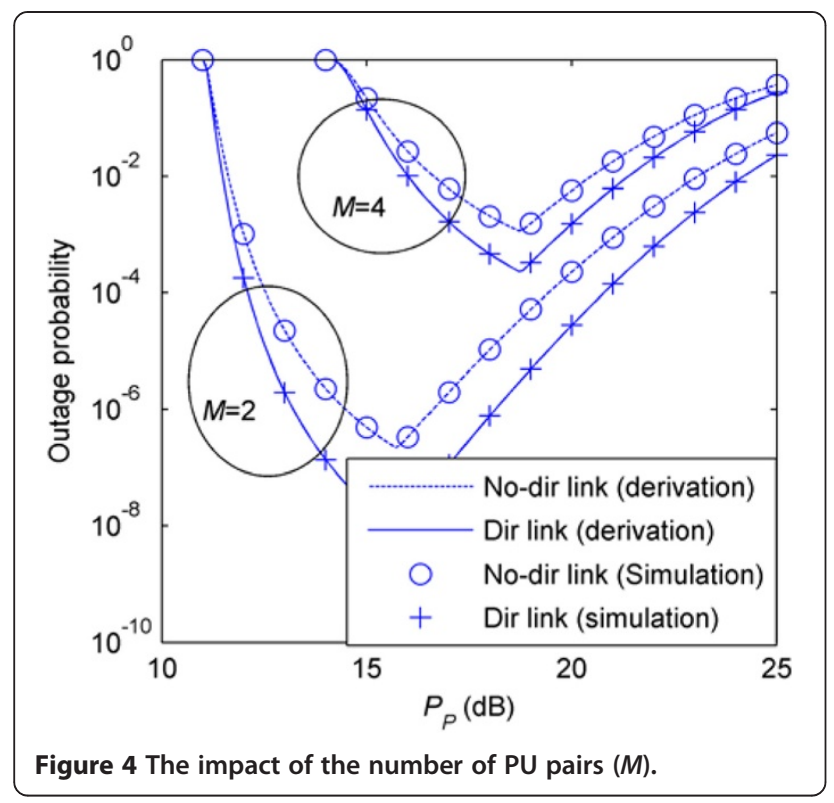

number of relays in the decoding subset DS is increased as the outage threshold $\mu_{\mathrm{th}}^{S}$ of the first hop is decreased. This yields that the outage performance of the UCR-OR systems is improved greatly and is dominated by the relay link. The gap is increasing with the number of relays in the decoding subset DS.

In Figure 4 , the impact of $M$ is investigated. In the figure, we take the mean power of cognitive direct link $\omega_{\phi}=0.5$, the outage constraint $\varepsilon=0.01$, and the outage threshold $\mu_{\text {th }}^{S}=\gamma_{\text {th }}^{S}=-10 d B$. The figure shows that the UCR-OR system with $M=2$ outperforms far the one with $M=4$.

In Figure 5, another aspect of the outage performance of the considered UCR-OR systems is presented. In the figure, by taking $\omega_{\beta_{S}}=\omega_{\beta_{R}}=\omega_{\alpha_{R}}=\omega_{\alpha_{D}}=0.6$, $P_{P}=14 d B, \varepsilon=0.01$, and $\mu_{\mathrm{th}}^{S}=\gamma_{\mathrm{th}}^{S}=-10 d B$, we present the outage probability versus the cognitive relaying link mean power $\omega_{g}\left(\omega_{g}=\omega_{h}\right)$. In Figure 5a, with $M=3$, the impact of the mean power of cognitive direct link is investigated. In the figure, we take $\omega_{\phi}=1,2$, and 3 $d B$, respectively. As obtained in Figure 2, it is found that the outage performance of the considered UCROR systems is improved as the mean power of direct link is increased. Besides this observation, we find that the slopes of outage probabilities are the same over the entire values of $\omega_{g}$. In Figure $5 \mathrm{~b}$, the impact of $M$ is investigated. It is easily seen that the value of $M$ has great impact on the outage performance. The result is similar as the one obtained in Figure 4.

By taking $P_{p}=14 d B, \lambda=1$, i.e., $P_{S}=P_{R}$, in Figure 6, we present the outage probability versus the transmit power $P_{s}$. Note that, in this case, the presented adaptive power scheme is not employed. Figure 6a,b shows that in high $P_{s}$, the asymptotic results match well with the exact ones, which corroborates the accuracy of our derivations. At the same time, Equations 60 and 61 also show that the diversity gain of secondary system is determined by the number of relays. The parameters of the primary system only affect the coding gain. These results are

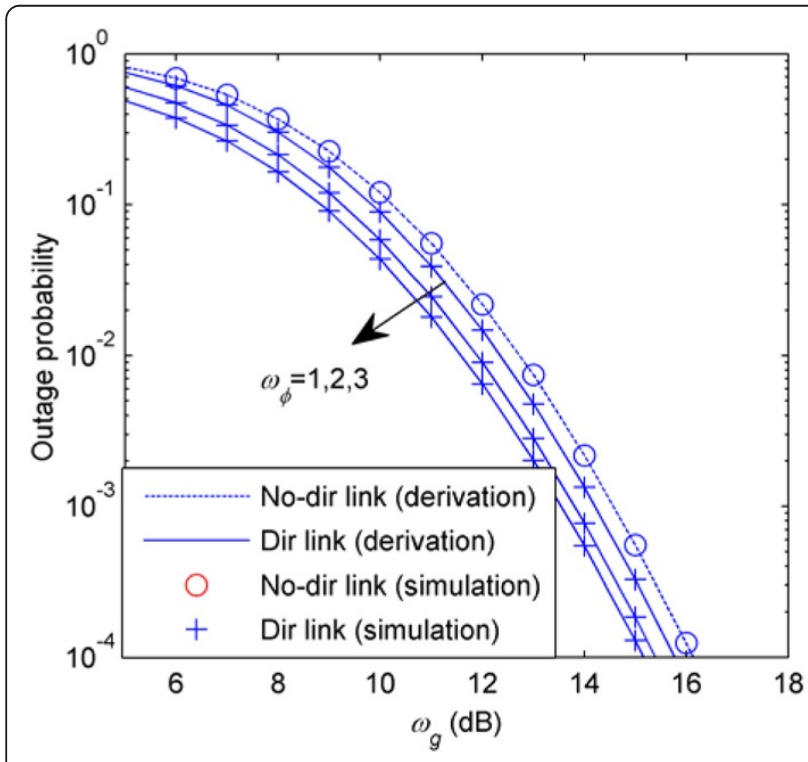

(a)

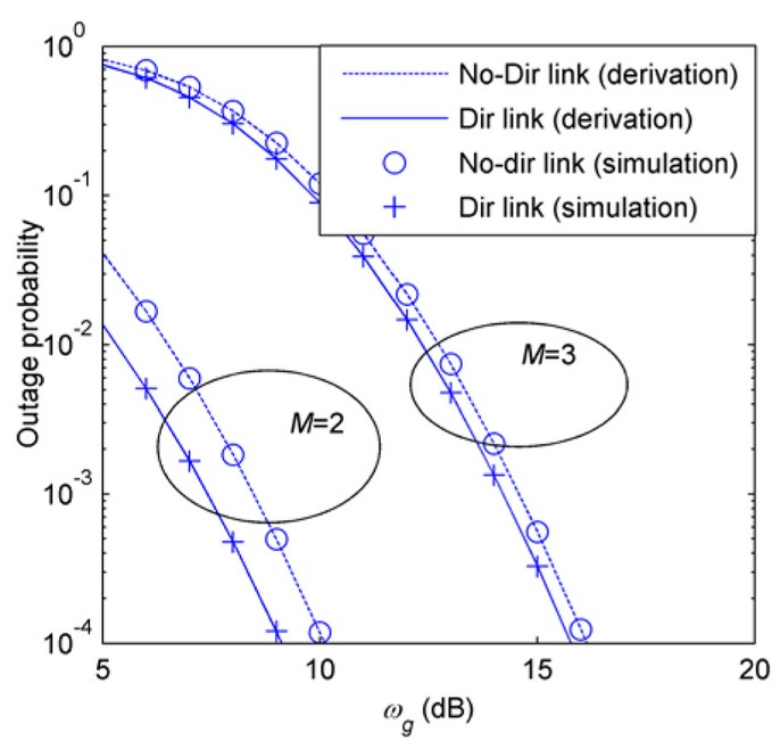

(b)

Figure 5 The outage probability versus the mean power $\boldsymbol{\omega}_{g}$. (a) Impact of $\omega_{\varphi}$ and (b) impact of $M$. 

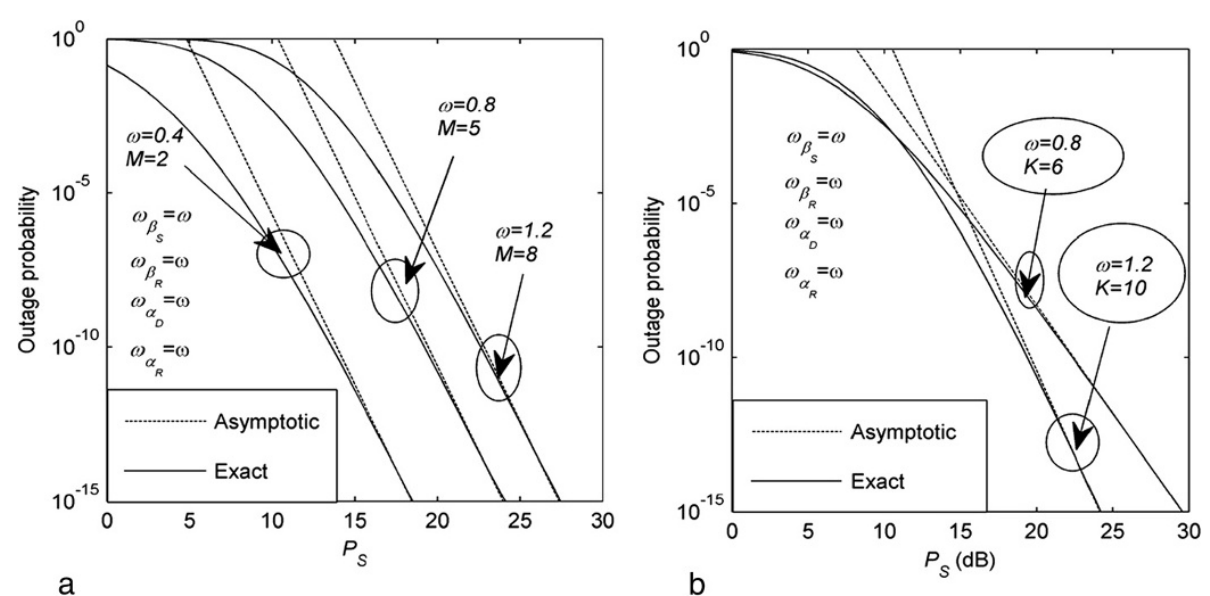

Figure 6 The asymptotic performance comparison analysis. (a) Fixed $K=10$ and (b) Variable $K(K=6,10)$. The arrows denote the corresponding curves for given system parameters.

validated by Figure 6a,b. For example, in Figure 6a, we take the fixed $K=10$. This yields that that the slopes of asymptotic outage probability are identical even if the parameters of primary system are different. In Figure 6b, the slopes of asymptotic outage performance are different. This is due to the fact that the values of $K$ are different.

\section{Conclusions}

In this work, we investigate the UCR-OR systems in terms of transmission power allocation and outage performance. Specially, we consider a system in which there are multiple primary user pairs and the direct path between cognitive source and destination. Under primary outage constraint and cognitive peak transmit power limit, the adaptive power allocation schemes for secondary users are achieved firstly. Then, we obtain the exact closed-form expression to the outage probability of UCR-OR systems with direct path transmission and multiple PUs' interference. Finally, to obtain the insight into the impact of system parameters on the performance of UCR-OR systems, by using the approximation of the high transmit power of SUs, the asymptotic closed-form expression of outage probability is achieved. The asymptotic results show that the diversity gain of the considered UCR-OR systems is determined by the number of relays. The parameters of primary systems only affect the coding gain but not the diversity gain. Simulated results validate the derivations firstly. At the same time, we investigate the impact of system parameters on outage performance such as the peak power $P_{P}$ of primary users, the mean power $\omega_{\phi}$ of cognitive direct path, and the number $M$ of primary user pairs. Specially, for the impact of $\omega_{\phi}$ and $M$, the simulations show that the direct path transmission can improve the performance of UCR-OR systems, and the number of primary users has very severe impact on the UCR-OR system's performance.

\section{Competing interests}

The authors declare that they have no competing interests.

\section{Acknowledgements}

The authors would like to thank the editors and the anonymous reviewers for their constructive comments and suggestions, which helped to improve the quality of this paper. This work was supported by the Natural Science Foundation of China under Grant 61261015, the 973 project 2013CB329104, the Natural Science Foundation of China under Grant 61372124, 61363059, and 61363059, the projects BK2011027, the Natural Science Foundation of Gansu Province for Distinguished Young Scholars (1308RJDA007), the China Postdoctoral Science Foundation 2012 M521105, and by the project 11KJA510001.

\section{Author details}

'College of Computer Science and Engineering, Northwest Normal University, Lanzhou 730070, China. ${ }^{2}$ Wireless Communication Key Lab of Jiangsu Province, Nanjing University of Posts and Telecommunications, Nanjing 210003, China. ${ }^{3}$ Key Lab of Broadband Wireless Communication and Sensor Network Technique of Ministry of Education, Nanjing University of Posts and Telecommunications, Nanjing 210003, China.

Received: 3 October 2013 Accepted: 14 July 2014 Published: 23 July 2014

\section{References}

1. JM Peha, Approaches to spectrum sharing. IEEE Commun. Mag. 43(2), 10-12 (2005)

2. L Dong, Performance analysis of MRC diversity for cognitive radio systems. IEEE Trans. Veh. Technol. 61(2), 849-853 (2012)

3. S Arkoulis, E Anifantis, V Karyotis, S Papavassiliou, N Mitrou, Discovering and exploiting spectrum power correlations in cognitive radio networks: an experimentally driven approach. EURASIP J. Wireless Commun. Netw. (2014). doi:10.1186/1687-1499-2014-17

4. S Shalmashi, SB Slimane, Performance analysis of relay-assisted cognitive radio systems with superposition coding, in Proc. of Personal Indoor and Mobile Radio Communications (PIMRC), 2012 IEEE 23rd International Symposium on, 2012, pp. 1226-1231 
5. HA Suraweera, PJ Smith, M Shafi, Capacity limits and performance analysis of cognitive radio with imperfect channel knowledge. IEEE Trans. Veh. Technol. 59(4), 1811-1822 (2010)

6. B Maham, P Popovski, Z Xiangyun, A Hjorungnes, Cognitive multiple access network with outage margin in the primary system. IEEE Trans. Wirel. Commun. 10(10), 3343-3353 (2011)

7. Z Caijun, T Ratnarajah, W Kai-Kit, Outage analysis of decode-and-forward cognitive dual-hop systems with the interference constraint in Nakagami-m fading channels. IEEE Trans. Veh. Technol. 60(6), 2875-2879 (2011)

8. S Stotas, A Nallanathan, On the throughput and spectrum sensing enhancement of opportunistic spectrum access cognitive radio networks. IEEE Trans. Wirel. Commun. 11(1), 97-107 (2012)

9. X Minghua, S Aissa, Cooperative AF relaying in spectrum-sharing systems: outage probability analysis under co-channel interferences and relay selection. IEEE Trans. Commun. 60(11), 3252-3262 (2012)

10. C Chinh, P Hoc, HJ Zepernick, Amplify-and-forward relay assisting both primary and secondary transmissions in cognitive radio networks over Nakagami-m fading, in Proc. of Personal Indoor and Mobile Radio Communications (PIMRC), 2012 IEEE 23rd International Symposium on, 2012, pp. 932-937

11. V Asghari, S Aissa, Performance of cooperative spectrum-sharing systems with amplify-and-forward relaying. IEEE Trans. Wirel. Commun. 11(4), 1295-1300 (2012)

12. X Jia, L Yang, Upper and lower bounds of two-way opportunistic amplify-andforward relaying channels. IEEE Commun. Lett. 16(8), 1180-1183 (2012)

13. X Jia, L Yang, H Fu, Tight performance bounds for two-way opportunistic amplify-and-forward wireless relaying networks with TDBC protocols. EURASIP J. Wireless Commun. Netw. (2011). doi:10.1186/1687-1499-2011-192

14. X Jia, H Fu, L Yang, L Zhao, Superposition coding cooperative relaying communications: outage performance analysis. Int. J. Commun. Syst. 24(3), 384-397 (2011)

15. JN Laneman, GW Wornell, Distributed space-time-coded protocols for exploiting cooperative diversity in wireless networks. IEEE Trans. Inform. Theory 49(10), 2415-2425 (2003)

16. K Junsu, DS Michalopoulos, R Schober, Diversity analysis of multi-user multirelay networks. IEEE Trans. Wirel. Commun. 10(7), 2380-2389 (2011)

17. HA Suraweera, PJ Smith, NA Surobhi, Exact outage probability of cooperative diversity with opportunistic spectrum access, in Proc. of Communications Workshops, 2008. ICC Workshops '08. IEEE International Conference on, 2008, pp. 79-84

18. G Xinrong, Y Weiwei, C Yueming, Outage performance of statistical CS assisted cognitive relay with interference from primary user. IEEE Commun. Lett. 17(7), 1416-1419 (2013)

19. W Xu, Z Jianhua, Z Ping, C Tellambura, Outage probability of decode-and-forward cognitive relay in presence of primary user's interference. IEEE Commun. Lett. 16(8), 1252-1255 (2012)

20. S JiangBo, L Zan, H HaiYan, C JunJie, G Rui, Capacity analysis of cognitive relay networks with the PU's interference. IEEE Commun. Lett. 16(12), 2020-2023 (2012)

21. H HaiYan, L Zan, S JiangBo, G Rui, Outage analysis of underlay cognitive multiple relays networks with a direct link. IEEE Commun. Lett. 17(8), 1600-1603 (2013)

22. TQ Duong, Y Phee-Lep, B Quoc, M Elkashlan, Y Nan, Cognitive relay networks with multiple primary transceivers under spectrum-sharing IEEE Signal Process Lett. 19(11), 741-744 (2012)

23. W Qihui, Z Zongsheng, W Jinlong, Outage analysis of cognitive relay networks with relay selection under imperfect CSI environment. IEEE Commun. Lett. 17(7), 1297-1300 (2013)

24. C Yan, VKN Lau, Z Shunqing, Q Peiliang, Protocol design and delay analysis of half-duplex buffered cognitive relay systems. IEEE Trans. Wirel. Commun. 9(3), 898-902 (2010)

25. HA David, HN Nagaraja, Order statistics, 3rd edn. (John Wiley, New York, 2003)

26. IS Gradshteyn, IM Ryzhik, Table of integrals, series, and products, 7th edn (Acdemic, San Diego, CA, 2007)

doi:10.1186/1687-1499-2014-122

Cite this article as: Jia et al:: Adaptive power allocation and outage performance of cognitive best relay cooperation systems with multiple primary transceiver pairs and direct path between cognitive source and destination. EURASIP Journal on Wireless Communications and Networking 2014 2014:122.

\section{Submit your manuscript to a SpringerOpen ${ }^{\circ}$ journal and benefit from:}

- Convenient online submission

- Rigorous peer review

- Immediate publication on acceptance

- Open access: articles freely available online

- High visibility within the field

- Retaining the copyright to your article

Submit your next manuscript at $>$ springeropen.com 